\title{
X-ray of Primary Site
}

National Cancer Institute

\section{Source}

National Cancer Institute. X-ray of Primary Site. NCI Thesaurus. Code C137928.

An X-ray of the primary site. 